which was crucial for the first-line doctors in evaluating the adequacy of medical resource.

Methods: We retrospectively analyzed the information of traumatic patients admitted to West China Hospital during the Lushan earthquake from April 20-27, 2013. Thirteen variables were extracted for the analysis, including gender, age, crush injury, multiple injury, injured time, injured places (in rooms or out), rescued ways (by oneself or others), transferred vehicles (ambulance or others), debridement, white blood cells counts, neutrophilic percentage, hemoglobin, and infection. Univariate analysis was conducted to compare the differences of those indicators mentioned above between the infected and uninfected patients. Moreover, multiple logistic regression analysis was performed to identify the factors associated with the complication of infection.

Results: There were 260 victims included in the present study, 90 of whom got infection, with the infection rate at $34.62 \%$. The univariate analysis showed that age and admission to ICU between the infected and uninfected victims are significantly different. The multiple logistic regression analysis indicated that crush injury, hemoglobin, and transferred vehicle had significant correlation with complication of infection, with their odds ratio being $1.482,0.987$, and 0.660 , respectively.

Conclusion: This study demonstrated that victims with crush injury or (and) low hemoglobin are more prone to complicate with infection during the inpatient period. This provides the evidence for doctors to decide which patients need debridement carefully, and more attendance as early as triage, in order to reduce the complication of infection and improve the efficiency of medical resource allocation after the earthquake.

Prehosp Disaster Med 2017;32(Suppl. 1):s38-s39

doi:10.1017/S1049023X17001182

\section{Emergency Health Care Demand}

Gerry J. Fitzgerald ${ }^{1}$, Sam Toloo ${ }^{1}$, Joanna Rego ${ }^{1}$, Peter Aitken ${ }^{2}$

1. School Of Public Health And Social Work, Queensland University of Technology, Brisbane/QLD/Australia

2. College Of Public Health, Medical And Veterinary Sciences, James Cook University, Townsville/QLD/Australia

Study/Objective: The five year Emergency Health Services Queensland (EHSQ) study aimed to describe the trends in EHS demand, to identify the factors driving increased demand, and to evaluate strategies which may safely reduce the future demand growth.

Background: Emergency health is a critical component of Australia's health system which has been fundamentally reformed over the last four decades. While these changes have improved the standards and quality of care, emergency health services are increasingly congested from the combined impact of growing demand and blocked access to inpatient care. This congestion has proven adverse to clinical, organizational, and staff impacts. However, the public, bureaucratic, and political perspective is that this problem somehow reflects at worst, gaming of the system, and so public policy solutions have been underpinned by blaming someone else for the problem. The feasible alternative proposition is that there are more sick people seeking care and exercising thoughtful and wise choices of the most appropriate source of that care.
Methods: This research used a mixed-methods approach comprising of analysis from the Queensland wide operational data, and interviews with 911 patients attending public hospital EDs.

Results: Our findings suggest that demand is growing across all developed nations; amongst the more urgent categories of patients, across all age groups, and a broad range of clinical conditions. The ultimate question underpinning this research is "can anything be done to moderate the growing demand while still offering safe, appropriate, and efficient care?"

Conclusion: Our analysis of remedial strategies has identified three broad categories:

\section{Enhanced primary and secondary prevention. Management on location.}

Diversion to alternative services including appropriate primary care services. The analysis of policy options has confirmed there is no single, or even small group of, interventions likely to make a difference. One distinct policy alternative is that the current arrangements offer the most efficient and effective means of providing the required care, and so ongoing expansion of those services may be most appropriate. However, the alternative is a comprehensive suite of strategies properly coordinated at both policy and operational levels, to ensure patients have access to a range of services from which they may choose the most appropriate to their needs, after weighing up the social, clinical, and financial implications. Additional research is required to develop and evaluate such an approach including the economic, clinical, professional, and social costs and benefits.

Prehosp Disaster Med 2017;32(Suppl. 1):s39

doi:10.1017/S1049023X17001194

Improvement in Success Rate of Intubation, Done by NonAnesthetist Emergency Physician with the Implementation of Rapid Sequence Intubation (RSI) Protocol in the

Emergency Department

Sanjeev Bhoi, Tej P. Sinha

Emergency Medicine, Jpn Apex Trauma Centre, All India institute of Medical Sciences, New Delhi/India

Study/Objective: The aim of this retrospective study was to determine whether the current practice of protocolised Rapid Sequence Intubation (RSI) has improved the success rate of intubation done by EP in ED of All India Institute of Medical Sciences (AIIMS) Trauma Center.

Background: Rapid Sequence Intubation (RSI) is key for securing airways in Emergency Departments (EDs). Literature on safety and feasibility of RSI by Emergency Physicians (EP) from India is limited.

Methods: It is a retrospective cohort study conducted in AIIMS, Level I Trauma Center. Data was collected on various parameters using primary information from the Red Area Patients Registry and Computerized Patient Record System (CPRS) of the period from November, 2007 to July, 2013. "Red area" is the specific area of the emergency department where emergency intubations are performed in cases of severely injured patients.

Results: Out of 2,117 intubations EP performed, $89.4 \%$ in pre-RSI group vs $97.9 \%$ in post RSI group. Anaesthetists 
intubated $10.6 \%$ pre-RSI group vs $2.1 \%$ post-RSI group. The compliance improved to $97.8 \%$ in overall intubations. Intubation rates among EP in pre RSI group vs post-RSI group in head injury, spine injury, shock and threatened airway were (90.4\% vs $98.3 \%),(71.4 \%$ vs $94.4 \%),(85.5 \%$ vs $97.4 \%)$ and (89.3\% vs $98.2 \%)$ respectively.

Conclusion: Emergency Physicians of non-anaesthetist training can perform rapid sequence intubation with comparable success rate in trauma patients.

Prehosp Disaster Med 2017;32(Suppl. 1):s39-s40

doi:10.1017/S1049023X17001200

Selected Immunological Indicators in the Assessment of, Risk of Severe Complications after Major Trauma

Aneta Binkowska ${ }^{1}$, Grzegorz Michalak ${ }^{2}$, Stawomir Pilip ${ }^{3}$, Robert Stotwinski ${ }^{4}$

1. Department Of Emergency Medicine, Department Of Medicine Of Disaster, Medical University of Warsaw, Warsaw/Poland

2. Department Of Emergency Medicine, Medical University of Warsaw, Warsaw/Poland

3. Medical Emergency Department, Medical University of Warsaw, WARSAW/Poland

4. Department Of Immunology, Biochemistry and Nutrition Medical University of Warsaw, Warsaw/Poland

Study/Objective: The aim of this study is to determine the immunological indicators, which depending on the extent of the injury, will have practical significance for the early diagnosis of severe complications after trauma.

Background: The pathophysiology of complications in patients with multi-organ injuries is still a subject of intense research.

Methods: The study reported in 32 patients admitted to the ER after trauma. Patients were divided into two groups, the group A ISS $\geq 20(n=20)$ and the group B ISS $<20(n=12)$. Laboratory tests and immunoassays were performed at the time of admission to the ER, and then repeated at 3, 6, 12, and 24 hours. The observation included clinical status of each patient, the incidence of complications, the type of treatments and mortality.

Results: In group A, 17 patients had complications (5 died), while in group B only 3 patients had complications. The most common complications included respiratory failure and infections. In our study, the highest level of IL-6 and IL-1Ra was recorded in 3 hours of acceptance in patients with late complications compared to the group without complications. Already in the 3 hour study, they could identify a group of people with a high risk of subsequent morbidity and mortality. The Receiver Operating Characteristic (ROC curve) analysis showed that the studied immune indicators can be prognostic markers of complications or death for patients after major trauma. In the groups of patients there was no significant difference for age, gender and basic laboratory diagnostics.

Conclusion: Our findings suggest that elevated levels of cytokines tested, a short time after the injury, may have a significant relationship with the occurrence of serious complications later. The diagnosis of early inflammatory response to injury should have an impact on the therapeutic. In addition to basic treatment, a potential target for therapeutic intervention should be taken into consideration; early cytokine response within the first 3 hours of hospitalization.

Prehosp Disaster Med 2017;32(Suppl. 1):s40

doi:10.1017/S1049023X17001212

Differences in the Treatment Outcomes of Referred and Non-referred Patients at the Accident and Emergency Department (AED) of Komfo Anokye Teaching Hospital (KATH)

Sonia Cobbold ${ }^{1}$, Paa Kobina Forson ${ }^{1}$, Joseph Bonney ${ }^{1}$, Evans X. Amuzu ${ }^{2}$

1. Accident And Emergency Department, Komfo Anokye Teaching Hospital, Kumasi/Ghana

2. Research And Development Unit, Komfo Anokye Teaching Hospital, Kumasi/Ghana

Study/Objective: This study sought to ascertain whether there is a difference in the characteristics and outcomes of patients of these categories - as measured by final triage color, health financing, mode of transportation to the AED, and treatment outcome (discharged home, transferred out to Main wards, or died).

Background: The Accident and Emergency Department (AED) of Komfo Anokye Teaching Hospital (KATH) is the main entry point for patients requiring critical care. Patients are either referred from other institutions or come on their own to the facility. The receiving triage room assesses vital signs, triages patients using the Triage Early Warning Score (TEWS), and assigns patients to the appropriate care center - utilizing Yellow ward (urgent management), Orange ward (very urgent management), and Red ward (emergency management). However, the outcomes of these referred and not referred patients are speculated to differ due to the consequences of delay in diagnosis, treatments, etc.

Methods: A prospective, cross-sectional study was employed at the Accident and Emergency center at the Komfo Anokye Hospital for six weeks. Data were collected and entered into an Epi information software database, exported, and analyzed using SPSS. Relation between variables was tested using ANOVA and correlation tests.

Results: There was no significant difference $(0.456)$ in the treatment outcomes of referred and non-referred patients. Hence, irrespective of how patients came to the hospital for treatment, treatment outcome (discharged home, Trans out to Main wards, or died) will not significantly change. Furthermore, there was no relation between status of patients (referred and non-referred) and by outcome of patients, final triage color, health financing, mode of transportation, type of admission, and drugs ordered.

Conclusion: There are no significant differences or relations in the treatment outcomes of referred and non-referred patients reporting to the Accident and Emergency Department (AED) of Komfo Anokye Teaching Hospital (KATH).

Prehosp Disaster Med 2017;32(Suppl. 1):s40

doi:10.1017/S1049023X17001224 